\title{
Isolation and Quantification of Extracellular DNA from Biofluids
}

\author{
L'ubica Janovičová1, Barbora Konečná1, Barbora Vlková1 and Peter Celec ${ }^{1,2,3, *}$
}

\author{
${ }^{1}$ Institute of Molecular Biomedicine, Faculty of Medicine, Comenius University, Bratislava, Slovakia; \\ ${ }^{2}$ Institute of Pathophysiology, Faculty of Medicine, Comenius University, Bratislava, Slovakia; \\ ${ }^{3}$ Department of Molecular Biology, Faculty of Natural Sciences, Comenius University, Bratislava, \\ Slovakia \\ *For correspondence: petercelec@gmail.com
}

\begin{abstract}
[Abstract] Extracellular DNA is studied as a diagnostic biomarker, but also as a factor involved in the pathophysiology of several diseases due to its pro-inflammatory properties. Extracellular DNA can be extracted from plasma, urine, saliva or other biofluids using standard DNA isolation procedures and specialized commercial kits. Sample preparation for isolation is important, freezing and thawing may affect the amount of extracellular DNA extracted. Subsequent centrifugations remove cells and cell debris from the samples to obtain true extracellular DNA. Small volume of samples especially from animal experiments is often an issue and it affects the DNA yield. Very short fragments ( $<100 \mathrm{bp})$ can be lost during isolation and are difficult to quantify using PCR. Fluorometric methods asses all stained DNA fragments. Selecting the method for quantification of extracellular DNA is crucial and combination of at least two methods is ideal. Standardization of procedures or at least their reporting in research papers is of utmost importance for comparison of results.
\end{abstract}

Keywords: Cell-free DNA, Circulating plasma DNA, Cell-free nucleic acids, DNA extraction, Sample preparation

[Background] Extracellular DNA often called cell-free DNA is a term for all DNA found outside of cells especially in diagnostic biofluids. Blood plasma DNA was first discovered by Mandel and Metais (1948). The interest in extracellular DNA research was stimulated much later by the research of the so-called liquid biopsy as a source of DNA-based biomarkers for non-invasive screening and diagnostics (Poon and Lo, 2001). The same extracellular DNA is, however, also involved in the pathophysiology of inflammatory diseases such as sepsis (Lauková et al., 2017), acute kidney injury (Jansen et al., 2017) and acute liver failure (Vokálová et al., 2017). The proinflammatory properties of extracellular DNA are in some of the sequences, especially mitochondrial DNA, resembling the sequences of bacterial DNA such as unmethylated $\mathrm{CpG}$ islands. In this case those proinflammatory properties are facilitated via detection with toll-like receptors. The nuclear DNA is more effective to trigger inflammation when associated with proteins such as histones or as a part of neutrophil extracellular traps. Extracellular DNA can be released from dying cells during apoptosis or necrosis. Most of the extracellular DNA in healthy individuals originates from blood elements.

The quantitative assessment of extracellular DNA is, thus, crucial for a wide range of research topics. There are many limitations when it comes to extracellular DNA measurement. In animal experiments, 
the available volume of blood, urine or saliva greatly limits subsequent analyses. Studies differ in the procedures used for sample collection, processing, isolation and quantification. This makes it impossible to use standard normal ranges for the concentration of extracellular DNA in plasma, urine or saliva. Generally, EDTA-containing tubes that inhibit the activity of deoxyribonucleases are mostly used for isolation of extracellular DNA from blood plasma (Lee et al., 2001). Sampling of urine or saliva is not standardized at all. Sample storage by freezing is common and often cannot be avoided. However, freezing and thawing can lead to unwanted degradation of extracellular DNA (Lahiri and Schnabel, 1993). Our unpublished observations suggest that isolation of DNA from fresh and frozen plasma leads to a completely divergent size profile of the extracellular DNA. Usually, two consecutive centrifugations are carried out to remove cells and smaller cellular debris from body fluids before DNA isolation. However, not all studies follow this procedure (Sun et al., 2018). Results from studies using different isolation and quantification methods cannot be compared. Due to high technical variability of the measurement of extracellular DNA concentrations the use of duplicates or triplicates could help achieve reproducible results. The standardisation of protocols for processing of body fluids intended for extracellular DNA isolation would be valuable for the research of extracellular DNA as a biomarker, but also as a damageassociated molecular pattern.

\section{Materials and Reagents}

1. Tubes for sample collection

a. Blood

Small volume: K3 EDTA microvettes (Sarstedt, catalog number: NC9990563)

Large volume: EDTA monovette (Sarstedt, catalog number: NC9453456)

b. Urine or saliva

Small volume: Eppendorf tubes (1.5 ml) (Eppendorf, catalog number: 022363204)

Large volume: Falcon tubes (15 ml) (Fisher Scientific, catalog number: 14-959-53A)

2. 96-well PCR plates (Thermo Fisher Scientific, catalog number: N8010560)

3. Adhesive PCR plate foils for 96-well PCR plates (Bio-Rad, catalog number: 1814030)

4. Thin-walled Qubit assay tubes $(0.5 \mathrm{ml})$ (Thermo Fisher Scientific, catalog number: Q32856) OR Black/white flat-bottom 96-well plates (Sigma-Aldrich, catalog number: CLS3925/CLS3922)

5. Primers (stored in the freezer at $-20^{\circ} \mathrm{C}$ )

a. Human nuclear DNA (F: 5'-gcttctgacacaactgtgttc-3', R: 5'-caccaacttcatccacgttca-3')

b. Human mitochondrial DNA (F: 5'-cataaaaacccaatccacatca-3', R: 5'-gaggggtggctttggagt-3')

6. $96 \%$ Ethanol (Fisher Scientific, catalog number: BP8202500)

7. Millipore water

8. Qubit dsDNA HS assay kit (Thermo Fisher Scientific, catalog number: Q32854) (stored in fridge at $4{ }^{\circ} \mathrm{C}$, used at room temperature)

9. QIAmp DNA Blood mini kit (Qiagen, catalog number: 51104) (stored at room temperature, protease after reconstitution stored in the fridge at $4{ }^{\circ} \mathrm{C}$ ) 
10. Sso Advanced Universal SYBR Green Supermix (Bio-Rad Laboratories, catalog number: 1725270) (stored in the freezer at $-20^{\circ} \mathrm{C}$ )

11. Agarose molecular grade (Sigma-Aldrich, catalog number: 05077)

12. TAE buffer (50x) (Thermo Fisher Scientific, catalog number: B49)

13. Loading dye (stored in fridge at $4{ }^{\circ} \mathrm{C}$ ) (Thermo Fisher Scientific, catalog number: R0611)

14. GoodView Nucleic Acid Stain (SBS Genetech, catalog number: HGV-2) (stored in fridge at $4{ }^{\circ} \mathrm{C}$ )

\section{Equipment}

1. Centrifuge with rotor suitable for falcon tubes and rotor for Eppendorf tubes

2. Real-time PCR cycler (QuantStudio ${ }^{\mathrm{TM}} 3$ Real-Time PCR System, 96-well, Thermo Fisher Scientific)

3. Qubit 3.0 fluorometer OR DeNovix QFX fluorometer OR fluorescence plate reader (Synergy H1, BioTek)

4. Vortex Genie 2 (Scientific Industries)

5. Multichannel Eppendorf pipette (8-channel, 30-300 $\mu \mathrm{l})$ (Eppendorf)

6. Electrophoresis

7. UV Transilluminator (UVP)

\section{Software}

1. Office excel (Microsoft) or any equivalent

2. Real-time PCR analysis software

3. Gen5 (Synergy H1 reader software; BioTek; available here)

\section{Procedure}

A. Sample processing (e.g., blood, urine, saliva, other body fluids)

1. Collect samples for the measurement of extracellular DNA into appropriate tubes. Collect blood in the EDTA-containing tube (purple). Collect urine and saliva into sterile Falcon tubes or Eppendorf tubes.

Notes:

a. The processing of samples is important and is the main source of technical variability. Serum collection tubes induce blood clotting and enable the release of DNA from blood cells during the coagulation process. Heparin-containing tubes prevent coagulation, but do not inhibit activity of deoxyribonucleases that are likely able to cleave extracellular DNA. EDTA inhibits activity of deoxyribonucleases and is the preferred anticoagulant. The ratio of anti-coagulant and blood should be consistent. This is easy to achieve in blood collection from human probands but can be difficult when blood from experimental animals is collected. 
b. Hemolysis increases the concentration of extracellular DNA in plasma. Hemolysed samples should be excluded from the final analysis of experiments. Both, stimulated and unstimulated saliva can be collected for the assessment of concentration of extracellular DNA. Saliva should be collected over the same amount of time, e.g., 10 min to assess saliva production. Urine with a low $\mathrm{pH}$ likely degrades extracellular DNA that is not protected.

2. Centrifuge samples at $1,600 \times g$ for 10 min at $4{ }^{\circ} \mathrm{C}$.

Note: Centrifugation of samples should be done immediately after sample collection. Samples should not be frozen before centrifugations to prevent additional cell lysis. Processing fresh samples may lead to different isolated DNA than using stored frozen samples. Ideally, samples should be processed without freezing. When analyzing fresh samples prolonged storage in fridge or at room temperature can also affect the DNA yield. Freezing and thawing of samples likely affects the integrity of vesicles containing DNA and complexes of extracellular DNA with proteins. In addition, samples should be warmed up to $37^{\circ} \mathrm{C}$ before isolation to decrease the viscosity and improve pipetting accuracy.

3. Pipette supernatant into a new Eppendorf tube. Be careful not to take cells in the pellet at the bottom of the tube.

Note: Pipetting of some types of samples can be harder after the first centrifugation. One needs to be mindful that plasma may not be completely separated, and pellet easily disturbed. Similarly, some saliva samples are more viscous than other. The issues with disturbing the pellet can be avoided by pipetting small volumes of liquid $(50 \mu l)$ and controlling the speed at which the mechanic pipette sucks up the liquid. This is especially true for low volume samples, e.g., mouse blood or saliva.

4. Centrifuge samples at $16,000 \times g$ for $10 \mathrm{~min}$ at $4{ }^{\circ} \mathrm{C}$. This centrifugation removes larger vesicles and apoptotic bodies from the samples.

5. After the second centrifugation there should be a small pellet visible at the bottom of the tube. Be careful not to take the pellet when pipetting into the new tube. Carefully pipette the supernatant into a new tube.

6. The supernatant can be used for the isolation of extracellular DNA using various commercially available kits.

B. Isolation of extracellular DNA (QIAmp DNA Blood mini kit manufacturers protocol)

1. Isolation is carried out according to the instructions in the protocol from the manufacturer.

2. Pipette $20 \mu \mathrm{l}$ of protease at the bottom of clean tube.

3. Add $200 \mu \mathrm{l}$ of sample. The volume can be adjusted depending on the sample material. Generally, urine contains very little extracellular DNA. The volume of sample can be increased.

4. Add $200 \mu \mathrm{l}$ of AL buffer.

5. Pulse-vortex the samples for $15 \mathrm{~s}$. Briefly centrifuge to remove droplets on the lid.

6. Incubate samples at $56^{\circ} \mathrm{C}$ for $10 \mathrm{~min}$. Briefly centrifuge samples again.

7. Add $200 \mu \mathrm{l}$ of $96 \%$ ethanol. Pulse vortex samples for $15 \mathrm{~s}$. 
8. Briefly centrifuge all samples again to remove droplets from the lids.

9. Pipette sample solution into spin columns included in DNA Blood mini kit.

10. Centrifuge samples at $6,000 \times g$ for $60 \mathrm{~s}$.

11. Discard the flow through in the small reservoir below column.

12. Add $500 \mu \mathrm{l}$ of AW1 solution to wash DNA bound to column.

13. Centrifuge at $6,000 \times g$ for $60 \mathrm{~s}$. Again, discard the flow through.

14. Add $500 \mu \mathrm{l}$ of AW1 solution.

15. Centrifuge at maximum speed, up to $20,000 \times g$ for $3 \mathrm{~min}$.

16. Discard the flow though and change the collection tube of column for a new one. Centrifuge again to remove residual wash buffers from column membrane.

Note: We highly recommend the optional centrifugation (Step B16). This helps to remove remaining buffer and ethanol from the column.

17. Transfer column into Eppendorf tube and add Millipore water to the column to elute the DNA. To concentrate the little extracellular DNA which is found in the samples use elution volume $50 \mu \mathrm{l}$ to $100 \mu \mathrm{l}$.

Note: The concentration of extracellular DNA is usually very low. Contamination introduced during sample processing and DNA isolation can, thus, easily affect the outcomes. To assess potential contamination DNA should be isolated also from a negative control, e.g., millipore water as one of the samples. After the isolation there might be traces of carrier DNA even in the negative control if measured using sensitive fluorometry. PCR should not be affected by this DNA traces. To minimize contamination sources, PBS should not be added as requested in the original DNA isolation protocol if sample volume is below $200 \mu$ l.

18. Centrifuge tubes with columns at $6,000 \times g$ for $60 \mathrm{~s}$.

19. After discarding the columns, tubes contain the isolated extracellular DNA.

C. Quantification of total extracellular DNA using single tubes (Qubit dsDNA HS assay kit, other fluorescence quantification options)

1. Prepare the working solution and mix it with samples as stated in the protocol of the manufacturer. Working solution can be premade by the manufacturer or prepared by mixing buffer and fluorescent dye (both provided by manufacturer). This depends on the kit which is used.

2. Avoid prolonged storage in light. Vortex the tubes but avoid measuring immediately after vortexing. Let the samples stand for at least one minute.

Note: Samples should not be measured right after vortexing because this leads to random spikes of fluorescence. It is important to mix the tubes well and avoid droplets sticking to the lid. Droplets can be removed by gentle shaking.

3. For the measurement the Qubit fluorometer or the DeNovix system can be used. Select correct settings on the reader. Select the correct kit which is used for the measurement on the reader. 
4. Measure standards. These are usually provided by kits. One standard being negative control (buffer or distilled water) and the high standard which contains known concentration of DNA.

5. Measure the fluorescence in samples and record the relative fluorescent units (RFU).

6. Measure samples 3 times to avoid errors (Note a). Calculate the concentration based on a calibration curve from the raw RFU values. Recalculate the concentration of DNA in samples based on the calibration curve, the used dilution and the elution volume (Note b).

Notes:

a. There are rarely errors displayed by the machine measuring the concentration of DNA. However, in some cases after prolonged storage DNA in high standard provided by manufacturer may degrade. It is important to note the RFU obtained throughout the time when using the machine and kit. Similarly, blank can be contaminated when repeatedly used. This can be prevented by aliquoting the standards.

b. The calibration curve is calculated in the same manner as one would calculate calibration curve for protein quantification. In this case, only two calibration points are used. Fluorescence has linear relationship with the concentration of DNA. Linear regression is used to recalculate concentration of DNA based on the RFU.

7. For the determination of sensitivity of measurement please see notes below.

Notes:

a. Sensitivity of the kit and measurements can be tested by determining limit of detection and limit of quantification. In most of cases, these are already provided by kit manufacturer as the suggested rage where fluorescence and DNA have linear relationship. The limit of detection is calculated by multiplying standard deviation of the blank by 3 , and the limit of quantification is calculated by multiplying standard deviation of the blank by 10 . This also applies to testing sensitivity of measurement using plate reader (Procedure D).

b. If we wanted to test it sensitivity further, we could prepare for example, five 10-fold dilution from DNA with starting concentration of $1,000 \mathrm{ng} / \mathrm{ml}$. This would provide us with tubes with $100 \mathrm{ng} / \mathrm{ml}, 10 \mathrm{ng} / \mathrm{ml}, 1 \mathrm{ng} / \mathrm{ml}, 0.1 \mathrm{ng} / \mathrm{ml}$ and $0.01 \mathrm{ng} / \mathrm{ml}$. If we would use the Qubit dsDNA HS assay kit we would be able to measure RFU higher thank blanks for first two dilutions. The dilutions of DNA with concentration $1 \mathrm{ng} / \mathrm{ml}, 0.1 \mathrm{ng} / \mathrm{ml}$ and $0.01 \mathrm{ng} / \mathrm{ml}$ would be at the level of blank.

D. Quantification of total extracellular DNA using a plate reader

1. Prepare a working solution based on the protocol of the manufacturer.

2. Prepare a calibration curve from the DNA standards and first test the sensitivity of the available fluorescence plate reader as this can vary considerably from instrument to instrument. Use white or black microplates to modulate sensitivity if needed.

Note: If measuring very low concentrations of extracellular DNA use a more sensitive kit that includes enhancers of fluorescence such as DeNovix ultra-high sensitivity kit or AccuBlue High 
sensitivity dsDNA kit. If measuring extracellular DNA in high concentration, e.g., from saliva, the less sensitive quantification options can be used.

3. Add samples to the working solution and mix by pipetting up and down several times with a multichannel pipette without forming bubbles.

Note: Slow careful pippeting up and down of samples helps to ensure that bubbles will not form. Bubbles can be prevented by avoiding the pipette tip to suck up air. Reverse pipetting or ideally dispensing can be used when pipetting working solution into wells.

4. Measure fluorescence using the fluorescence plate reader. The excitation wavelength is $485 \mathrm{~nm}$ and the emission wavelength is $530 \mathrm{~nm}$. Measure each plate at least three times to check the stability of the fluorescence in each well.

E. Quantification of nuclear and mitochondrial extracellular DNA using real-time PCR

1. Prepare PCR master mix for all planned reactions. Always include positive and negative controls. Note: The positive control is a template of either nuclear of mitochondrial DNA of known concentration. These can be obtained from isolation and PCR reaction. Concentration is determined using the fluorescence quantification methods, e.g., Qubit dsDNA HS assay kit. The serial dilutions can be prepared from the isolated sample. Purified PCR products can be sequenced to further prove the specificity and can be used as standards for the calibration curve. The resulting calibration should consist of 2-fold to 10-fold dilutions depending on the sample range which should be determined beforehand by analysing several samples. Negative control and diluent of the DNA is the molecular grade Millipore water.

2. Pipette PCR master mix, primers and samples in hood to avoid contamination.

Note: The PCR reaction can be decreased in volume if desired. It can be decreased in up to 15 to $10 \mu \mathrm{l}$. Different real-time master mixes can used for the quantification. There is need for the standardization of the assay. We have mentioned two master mixes which we have used, and they worked with this type of analysis.

3. Run the real-time PCR targeting single copy genes in nuclear and mitochondrial DNA. Sequences of suggested primers for human extracellular DNA can be found in the materials section. Be aware of the presence of mitochondrial sequences in the nuclear DNA.

4. Check the presence and absence of bands on gel electrophoresis to determine the specificity of the PCR (Note a). Troubleshooting options are discussed in Notes $b$ and $c$ below.

Notes:

a. Quantification of extracellular DNA using real-time PCR is a sensitive and powerful method. Contamination introduced in the process of isolation affects the final measured concentration of extracellular DNA. Ideally, all samples should be isolated at the same time with identical conditions. The real-time PCR should be done on all samples at the same time. Using triplicates can help to achieve reproducible results.

b. Further troubleshooting is sometimes necessary to achieve best results. The specificity and sensitivity of reaction are crucial. Usually, there is only few copies of DNA isolated from body 
fluids. Most of the DNA is in cells or vesicles which are removed in centrifugation. The free DNA fragments are typically short. Specificity of reaction can be assessed based on melting curves from real-time PCR and from gel electrophoresis. If primer dimers are present or melting curves do not have characteristic peak, reaction need to be adjusted. This is usually done by adjusting annealing temperature.

c. Sensitivity of reaction can be determined similarly as for the fluorescence measurement described in Step C7 Note b. The real-time PCR can detect DNA at low concentration, it is extremely sensitive. The detection of few copies of DNA are possible with this method.

\section{Acknowledgments}

The authors are supported by grants from the Slovak Research and Development Agency (APVV16-0273) and Ministry of Education of the Slovak Republic (VEGA 1/0092/17). The protocol for sample processing is based on the widely used method developed by Dennis Lo and colleagues described for example in Chiu et al. (2003).

\section{Competing interests}

No competing interests.

\section{Ethics}

All described procedures for sample collection were approved by the Ethics committee of the Institute of Molecular Biomedicine, Comenius University in Bratislava (ro-536/18-221/3; 01/07/201801/05/2020).

\section{References}

1. Jansen, M. P., Emal, D., Teske, G. J., Dessing, M. C., Florquin, S. and Roelofs, J. J. (2017). Release of extracellular DNA influences renal ischemia reperfusion injury by platelet activation and formation of neutrophil extracellular traps. Kidney Int 91(2): 352-364.

2. Lahiri, D. K. and Schnabel, B. (1993). DNA isolation by a rapid method from human blood samples: effects of $\mathrm{MgCl}_{2}$, EDTA, storage time, and temperature on DNA yield and quality. Biochem Genet 31(7-8): 321-328.

3. Lauková, L., Konecna, B., Babickova, J., Wagnerova, A., Meliskova, V., Vlkova, B. and Celec, P. (2017). Exogenous deoxyribonuclease has a protective effect in a mouse model of sepsis. Biomed Pharmacother 93: 8-16. 
4. Lee, T. H., Montalvo, L., Chrebtow, V. and Busch, M. P. (2001). Quantitation of genomic DNA in plasma and serum samples: higher concentrations of genomic DNA found in serum than in plasma. Transfusion 41(2): 276-282.

5. Mandel, P. and Métais, P. (1948). Les acides nucléiques du plasma sanguin chez l'Homme. Comptes Rendus des Seances de la Societe de Biologie et de ses Filiales. 142 (3-4): 241-243.

6. Poon, L. L. and Lo, Y. M. (2001). Circulating fetal DNA in maternal plasma. Clin Chim Acta 313(1-2): 151-155.

7. Sun, K., Jiang, P., Wong, A. I. C., Cheng, Y. K. Y., Cheng, S. H., Zhang, H., Chan, K. C. A., Leung, T. Y., Chiu, R. W. K. and Lo, Y. M. D. (2018). Size-tagged preferred ends in maternal plasma DNA shed light on the production mechanism and show utility in noninvasive prenatal testing. Proc Natl Acad Sci U S A 115(22): E5106-E5114.

8. Vokálová, L., Laukova, L., Conka, J., Meliskova, V., Borbelyova, V., Babickova, J., Tothova, L., Hodosy, J., Vlkova, B. and Celec, P. (2017). Deoxyribonuclease partially ameliorates thioacetamide-induced hepatorenal injury. Am J Physiol Gastrointest Liver Physiol 312(5): G457-G463. 